\title{
Primary Cardiac Angiosarcoma: A Case Report and Review of the Literature
}

\author{
Shiqi Guo ${ }^{1}$ and Qiang Guo ${ }^{1}$ \\ ${ }^{1}$ First Affiliated Hospital of Soochow University
}

August 7, 2020

\begin{abstract}
We reported a 34-year-old man who was diagnosed as primary cardiac angiosarcoma. The diagnosis was delayed until one month after he first entered the hospital. Because cardiac angiosarcoma is easy to be misdiagnosed as pneumonia or other diseases due to its rarity. Although surgical therapy is always considered as a method that can prolong survival time, highly malignant tumors with local infiltration and systemic metastasis lead to poor prognosis. We stressed that primary cardiac malignancy should be noticed when a young patient presented as unexplained pericardial effusion or refractory pneumonia. Early diagnosis can provide more possibilities for treatments.
\end{abstract}

\section{Keywords}

cardiac tumor, pericardial effusion, pneumonia, angiosarcoma, cardiac imaging

\section{Introduction}

Primary cardiac angiosarcoma is a rare tumor, but it is the most common histologic subtype in primary cardiac malignant tumor in adults ${ }^{1,2}$. Although advanced technologies have been applied to diagnose heart tumors, such as tumor markers, echocardiography, computer tomography $(\mathrm{CT})$, magnetic resonance imaging (MRI), and positron emission tomography/computer tomography (PET/CT), cardiac tumor are still easily misdiagnosed as other diseases at the onset of disease. Clinicians try everything possible such as surgical therapy and other comprehensive treatments to actively treat primary cardiac angiosarcoma. However, due to the highly aggressive of this disease, the patient's outcome is often poor ${ }^{2,3}$.

\section{Case representation}

A 34-years-old man was hospitalized due to repeated chest tightness, breathlessness, pericardial effusion and syncope, which happened once lasted 10 seconds. Based on the result of CT scan (Figure 1A), a large amount of pericardial effusion existed accompanied by multiple inflammation in bilateral lungs, so the patient was considered to be pneumonia. After the drainage of pericardiocentesis, chest tightness and breathlessness relived. But the pneumonia still persisted after being given adequate infection resistance aimed at pneumonia. Four weeks later, a similar situation occurred again. At the same time, the patient had intermittent tingling on the right chest, severe cough, and hemoptysis once. Based on the image of CT scan (Figure 1B), large amount of pericardial effusion and severe lung infection were noted. The specimen of pericardial puncture was sent to the laboratory. From the results of the chemical examination of pericardial effusion, a fragment of Cytokeratin 19 (CYFRA 21-1), ferritin, and cancer antigen 125 (CA125) escalated. Serum tumor markers were detected, indicating that CA125 and Tumor Supplied Group of Factors (TSGF) elevated. Echocardiography(Figure 2)and Contrast-enhanced chest CT scan (Figure 3) showed a mass located at the right atrioventricular junction. Contrast-enhanced chest CT confirmed filling 
defect in the right atrioventricular junction and some pleural effusion on both sides. Fluorodeoxyglucose18 combined positron emission tomography/computer tomography $\left({ }^{18} \mathrm{~F}-\mathrm{FDG}-\mathrm{PET} / \mathrm{CT}\right)$ (Figure 4) showed abnormal soft tissue lesions on the right anterior edge of the heart with increased focal glucose metabolism and increased focal radioactivity uptake, maximum standardized uptake values (SUVmax)=11.9, multiple patchy images of both lungs with uneven glucose metabolism. In conclusion, tumor markers were abnormal in the pericardial effusion and peripheral blood. According to the echocardiography, space-occupying of right atrium was observed. Moreover, ${ }^{18} \mathrm{~F}$-FDG-PET-CT showed a hypermetabolic lesion in the right atrium, so the possibility of cardiac malignancy was presumed. After informing the patient of the surgical risk, the surgery was preferred and the sampling was sent for pathological examination (Figure 5). The results showed that the tumor was composed of atypical endothelial cells with pathological mitotic images. Besides, the blood vessel channels composed of atypical endothelial cells were poorly formed and communicated with each other. Immunohistochemistry demonstrated: Vimentin(+), CD34(+), CD31(+), F11-1(+), CD31 and CD34 are highly sensitive and specific to vascular tumors ${ }^{4,5}$, so the diagnosis of primary cardiac angiosarcoma was clearly. However, the tumor was not removed completely and the pathological results showed that the surgical margin was still positive, which may lead a devastating blow to the patient's prognosis.

\section{Discussion}

Cardiac angiosarcoma is very likely to be misdiagnosed as pneumonia, since rarity of the disease and the non-specific clinical manifestations ${ }^{6}$, including the difficulty of breathing, chest pain, anemia, et cetera, determined by the size of the tumor and anatomical position ${ }^{7}$. Besides, primary cardiac angiosarcoma is highly invasive, with the characteristics of early systemic metastasis, and the lungs and pericardium are common sites of invasion. Therefore, the primary symptoms of tumors can be masked.

Angiosarcoma is the most common histologic subtype in primary cardiac malignant tumor among adults ${ }^{1,2}$, which usually occurs in men aged under 65 years, with a male to female ratio of around 2-3:1,6. The most vulnerable site is in the right atrium, for $74 \%$ of primary cardiac angiosarcoma occur in the right atrium and often interfere with adjacent structures ${ }^{2}$. The case we reported was an angiosarcoma located at the right atrioventricular junction of a 34-year-old male patient.

At present, advanced inspection technology have been applied in the detection of heart tumors, such as tumor markers, echocardiography, CT, MRI, and PET/CT. Above all, echocardiography valued as the most effective way for the test, but heart tissue biopsy is the golden standard ${ }^{2-5}$. In this case, it was the echocardiogram that prompted the abnormal position of the heart. Afterwards, the biopsy tissues we collected were positive in CD34, CD31, and F11-1; then primary cardiac angiosarcoma was diagnosed ${ }^{8,9}$.

Highly malignant tumors with local infiltration and systemic metastasis lead to poor prognosis of cardiac angiosarcoma. The survival time of the patients is only 5 to 13 months $^{2,3}$. The rarity of this diagnosis makes it hard to regulate standardized treatments. Studies have shown that neoadjuvant chemotherapy, local radical resection, postoperative radiotherapy, and targeted therapy may better prolong the survival rate $^{7,10,11}$. In this case, the tumor was not completely removed, that may lead a devastating blow to the patient's prognosis.

\section{Conclusion}

primary cardiac angiosarcoma is a rare tumor, which is easy to be misdiagnosed as pneumonia or other diseases. Patients always have an insufficient prognosis since its high aggressiveness and rapid progression. Additionally, the emphasis is probably not to charm away, but rather to put an appropriate diagnostic workup for primary cardiac angiosarcoma in the setting. Early diagnosis could prevent tumor progression and metastasis, even provide opportunities for further treatment.

\section{Reference}

1. Ambrus N, Havasi K, Kalapos A, et al. Primary cardiac angiosarcoma: A case report. Echocardiography. 2018;35(2):267-271. 
2. Liu C, Zhao Y, Yin Z, et al. Right atrial epithelioid angiosarcoma with multiple pulmonary metastasis confirmed by multimodality imaging-guided pulmonary biopsy: A case report and literature review.Medicine (Baltimore). 2018;97(30):e11588.

3. Yu JF, Cui H, Ji GM, et al. Clinical and imaging manifestations of primary cardiac angiosarcoma. $B M C$ Med Imaging. 2019;19(1):16.

4. Geller RL, Hookim K, Sullivan HC, Stuart LN, Edgar MA, Reid MD. Cytologic features of angiosarcoma: A review of 26 cases diagnosed on FNA. Cancer Cytopathol. 2016;124(9):659-668.

5. Patel SD, Peterson A, Bartczak A, et al. Primary cardiac angiosarcoma - a review. Med Sci Monit. 2014;20:103-109.

6. Kim J, Da Nam B, Hwang JH, et al. Primary cardiac angiosarcoma with right atrial wall rupture: A case report. Medicine (Baltimore).2019;98(14):e15020.

7. Yanagawa B, Chan EY, Cusimano RJ, Reardon MJ. Approach to Surgery for Cardiac Tumors: Primary Simple, Primary Complex, and Secondary. Cardiol Clin. 2019;37(4):525-531.

8. Rao U, Curtin J, Ryding A. Primary angiosarcoma of the heart.Heart. 2013;99(24):1878-1879.

9. Pazhenkottil AP, Bode PK. Angiosarcoma Involving the Heart. N Engl J Med. 2020;382(9):855.

10. Ramlawi B, Leja MJ, Abu Saleh WK, et al. Surgical Treatment of Primary Cardiac Sarcomas: Review of a Single-Institution Experience.Ann Thorac Surg. 2016;101(2):698-702.

11. Hoffmeier A, Sindermann JR, Scheld HH, Martens S. Cardiac Tumors.Deutsches Aerzteblatt Online. 2014.

Figure Legends

Figure 1. Chest CT scan in the 34-year-old man

A. Large amount of pericardial effusion and severe lung infection were noted on day 1.

B. Similar images four weeks later. Pericardial effusion was pointed by yellow arrows.

Figure 2. An Echocardiographic in the 34-year-old man

A. A mass (pointed by a yellow arrow) about $1.7 \mathrm{~cm} \times 4.2 \mathrm{~cm}$ was explored in the anterior wall of the right atrium with infiltration; and partly entered the anterior wall of the tricuspid annulus and the right ventricle.

B. A leaf-shaped loose mass (pointed by a yellow arrow) about $2.4 \mathrm{~cm} \times 3.1 \mathrm{~cm}$ was in right atrium, with irregular edges and floating with the blood flow. $\mathrm{RA}=$ right atrium; $\mathrm{RV}=$ right ventricle.

Figure 3. A contrast-enhanced chest CT scan in a 34-year-old man

About $2.5 \mathrm{~cm}$ sized a low-density mass is noted in the right atrium with a small amount of bilateral pleural effusions. Filling defect at right atrium was pointed by the arrow. $L V=$ left ventricle; $R A=$ right atrium; $\mathrm{RV}=$ right ventricle.

Figure 4. A ${ }^{18}$ F-FDG-PET/CT scan in the patient aged 34

A, B. Hypermetabolism is seen in the cardiac mass of the right atrium, maximum standardized uptake values was 11.9.

C. Multiple plaque shadows in lungs with uneven glucose metabolism.

Figure 5. Histology of biopsy from the right atrium mass

A. The tumor cells were obviously heteromorphic, round and pleomorphic (hematoxylin and eosin, $4 \times$ ). 
B. The tumor was composed of atypical endothelial cells with badly formed vascular channels, which was pointed by a black arrow (hematoxylin and eosin, $10 \times$ ).

C. Pathological mitotic image was pointed by a red arrow (hematoxylin and eosin, 40×).

Figure 6. Immunohistochemistry of the right atrium mass

A. Tumor cell cytoplasm Vimentin (+); B. Tumor endothelial cell membrane CD31(+); C. Tumor endothelial cell membrane CD34(+).
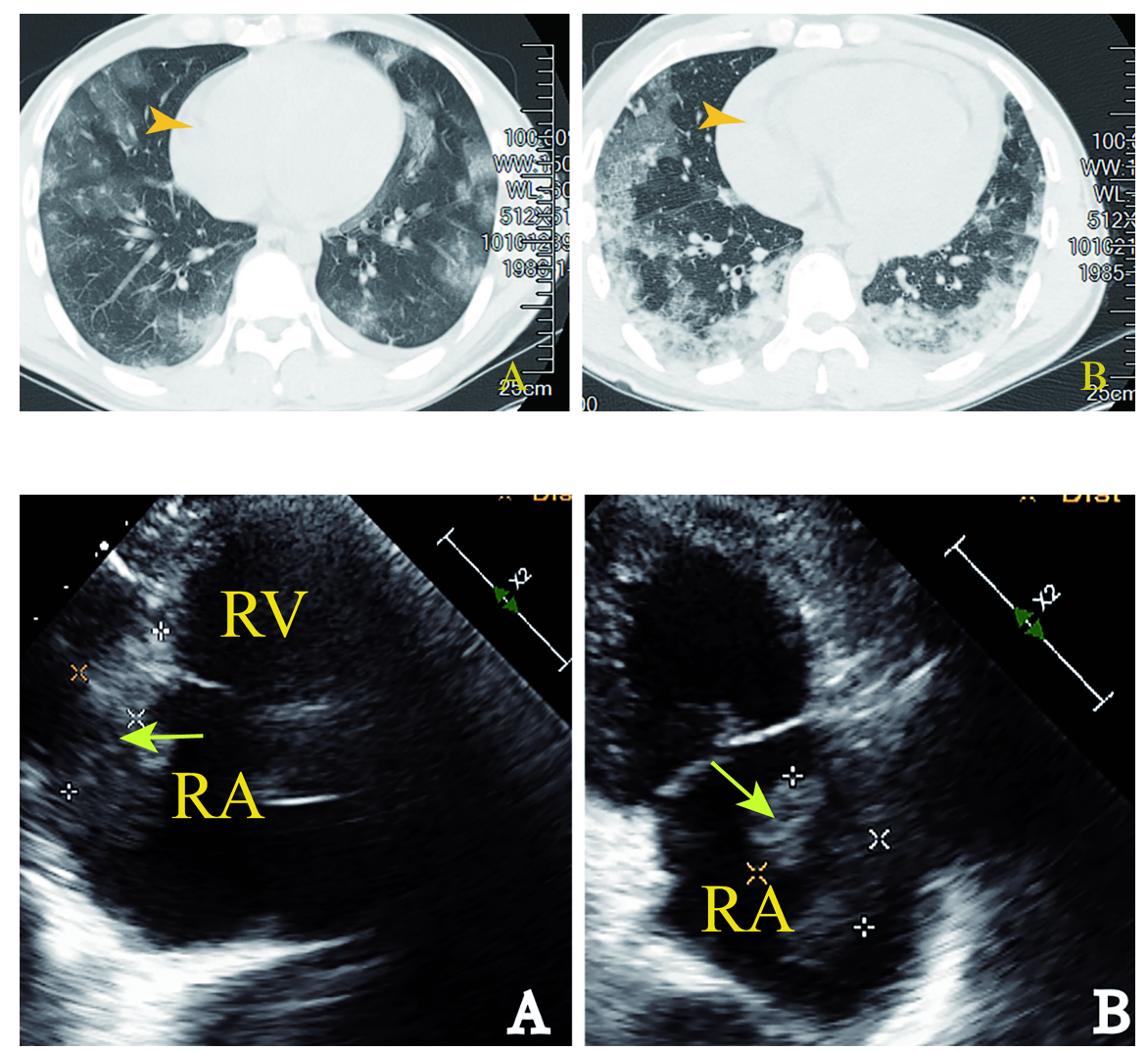

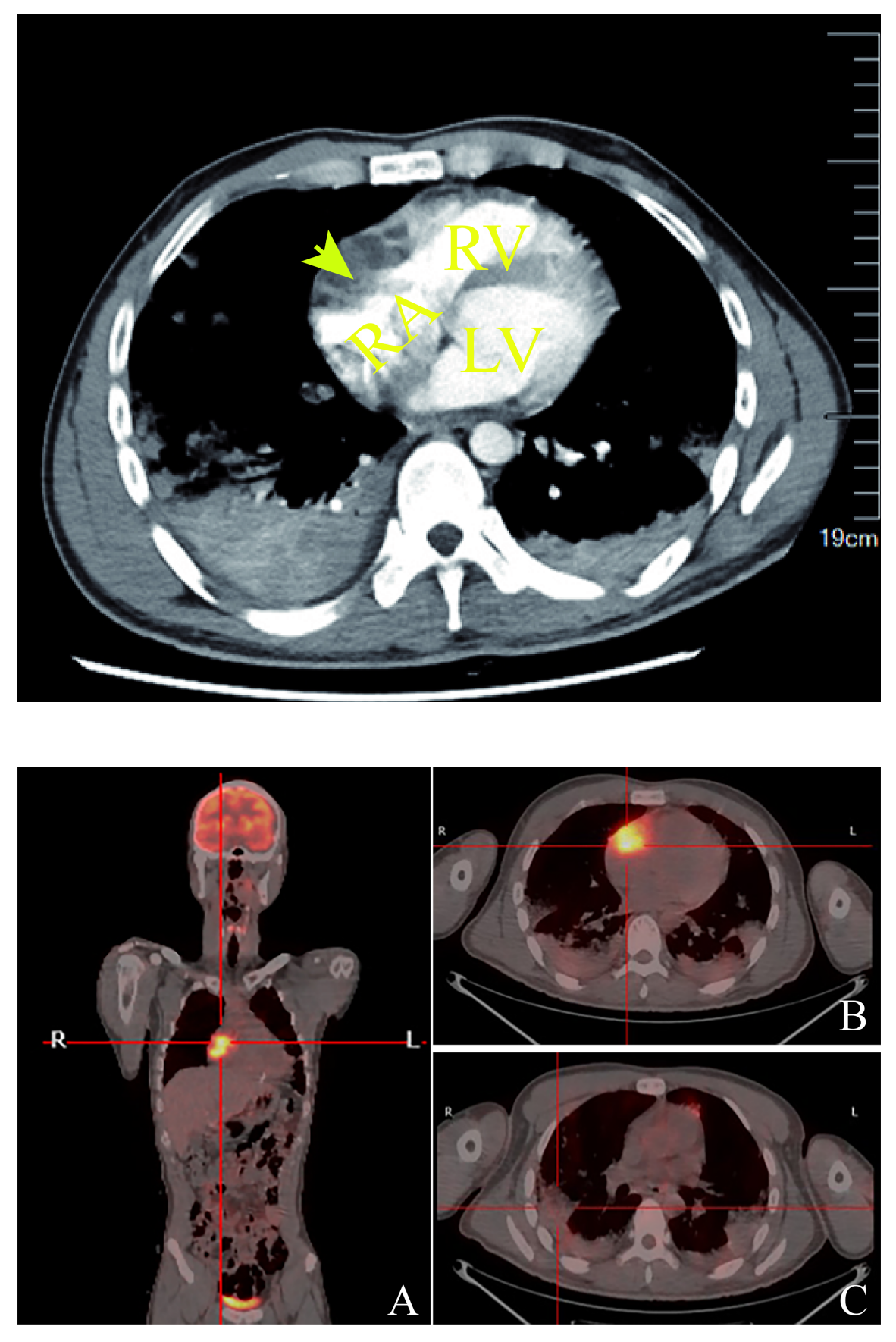

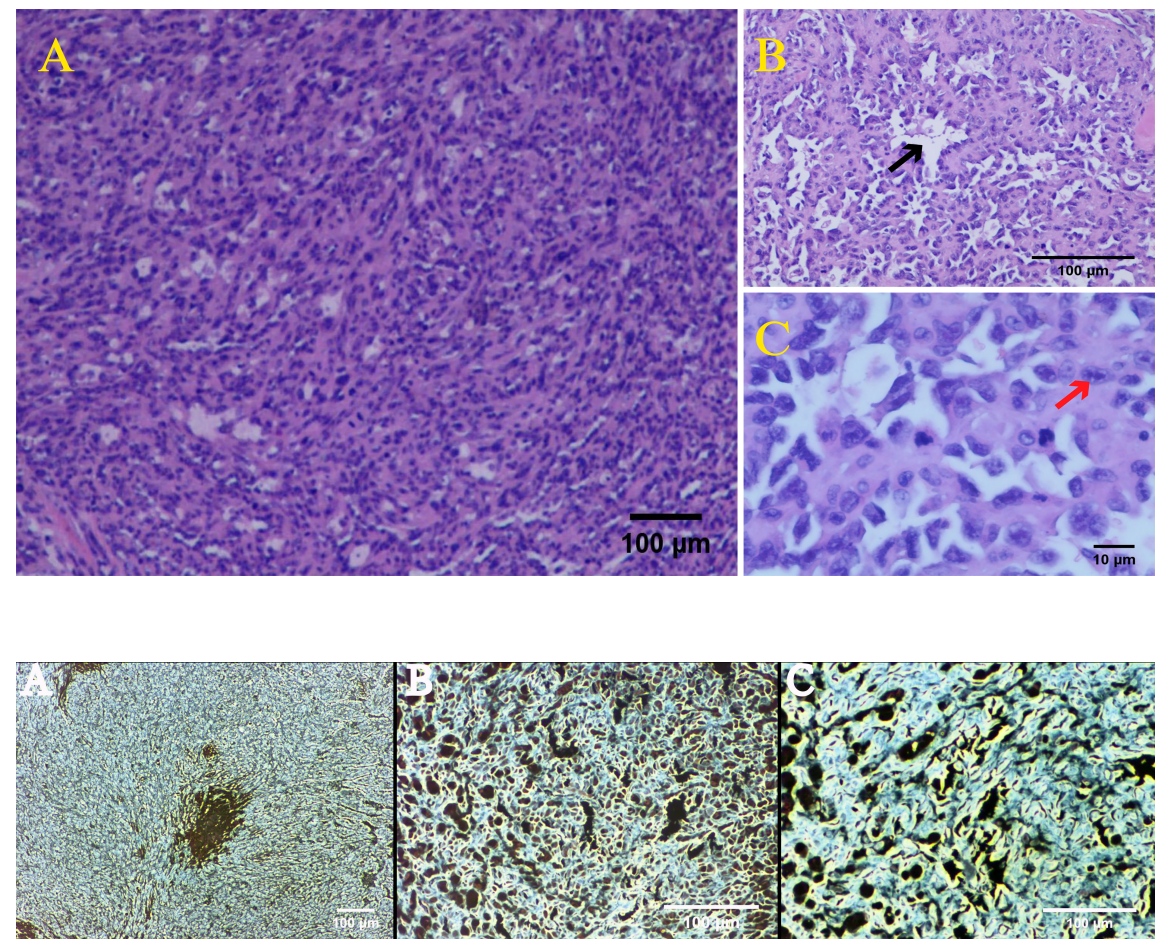